\title{
A Fatal Complication Caused by Occult Pheochromocytoma After Splenic Artery Embolization for Malignant Hypersplenism
}

\author{
Wouter Dinkelaar · Otto Elgersma • \\ Mark-David Levin
}

Published online: 22 December 2011

(c) The Author(s) 2011. This article is published with open access at Springerlink.com

\section{Introduction}

Splenic artery embolization (SAE) is a well-established method for treating traumatic splenic hemorrhage as well as splenic neoplasms [1-3]. This procedure may provide an alternative to open surgery. However, there are risks involved with SAE, mainly due to possible complications after the procedure. Most frequent complications are lactate acidosis, sepsis, renal insufficiency, and bowel perforation. We describe a patient with malignant hypersplenism who underwent SAE to treat severe thrombocytopenia. Complications several days after the procedure included bowel necrosis, probably caused by an occult pheochromocytoma and triggered by stress caused by the procedure.

\section{Case}

A 63-year-old man underwent SAE in order to improve his chronic severe thrombocytopenia-caused splenomegaly due to mantle cell lymphoma. The patient had a substantial medical history including coronary heart disease, for which he recently underwent a percutaneous intervention with

\footnotetext{
W. Dinkelaar $(\bowtie)$

Leiden University Medical Center,

Laan van Meerdervoort 208, 2517, BJ,

The Hague, The Netherlands

e-mail: wouterdinkelaar@hotmail.com

O. Elgersma - M.-D. Levin

Albert Schweitzer Hospital, Postbus 444,

3300, AK, Dordrecht, The Netherlands

e-mail: o.elgersma@asz.nl

M.-D. Levin

e-mail: m-d.levin@asz.nl
}

placement of several coronary stents, a recently discovered non-small cell lung carcinoma in the left lower lobe, and a stage IVa mantle cell lymphoma, for which he recently received high-dose chemotherapy. A computed tomography (CT) scan before SAE revealed extensive residual lymphadenopathy, severe splenomegaly (Fig. 1) with a sagittal diameter of more than $20 \mathrm{~cm}$, and a lung tumour with a diameter of $3 \mathrm{~cm}$. No abnormalities in the adrenal glands were noted.

At the time of SAE, the patient's complaints were angina pectoris New York Heart Association class I-II without night sweats, weight loss, or fever. Physical examination revealed a World Health Organization performance status of 1, blood pressure of $120 / 75 \mathrm{~mm} \mathrm{Hg}$, pulse of 70 beats/min, and a large spleen palpable $6 \mathrm{~cm}$ under the costal margins. A digital subtraction angiography with midstream abdominal aorta injections was performed via retrograde puncture of the right femoral artery. This revealed a massive spleen and a patent splenic artery originating from the celiac trunk. After selective catheterization (Fig. 2), the splenic artery just distal to the dorsal pancreatic artery origin was occluded with multiple coils (5-, 8-, and 10-mm 0.035-inch fibered platinum coils; Boston Scientific, Natick, MA). Selective contrast injections in the celiac trunk afterward demonstrated stasis of contrast in the splenic artery, indicating successful embolization (Fig. 3). The dorsal pancreatic artery was still patent. There were no signs of acute complications due to the intervention.

Several hours after the procedure, however, the patient developed abdominal pain in the left upper quadrant for which he received a total of $4 \mathrm{~g}$ of acetaminophen and 50 $\mathrm{mg}$ of tramadol upon request at $24 \mathrm{~h}$. At physical examination, his blood pressure was 170/115 mm $\mathrm{Hg}$ and his pulse 100 beats/min. Physical symptoms were mild abdomen pain at compression of the left upper quadrant without signs of peritonitis. Two days later, the patient was discharged in reasonable condition with mild abdominal pain. 


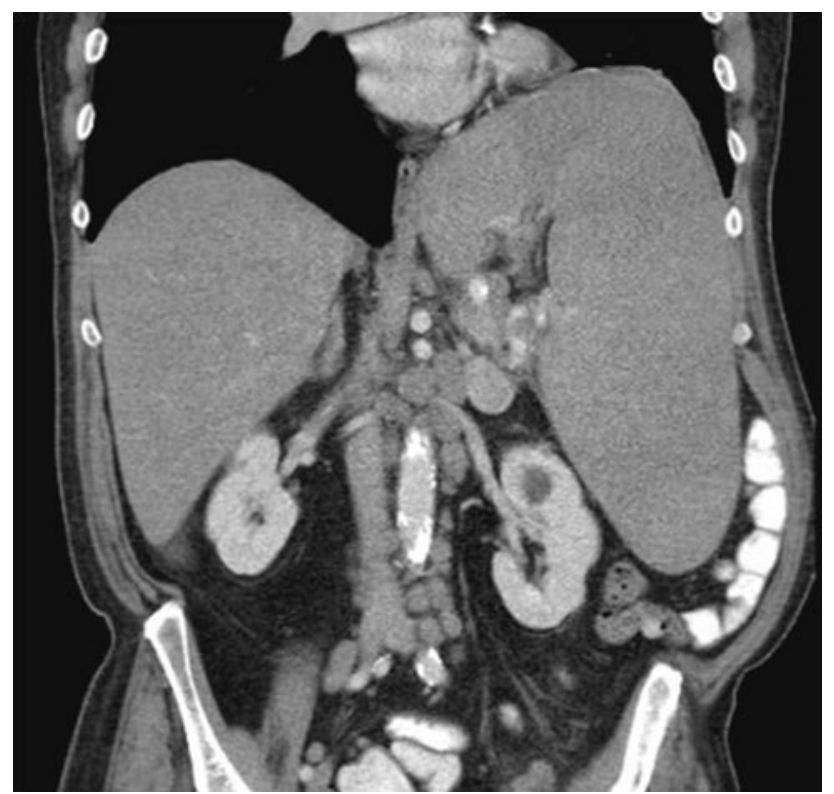

Fig. 1 Severe splenomegaly before SAE

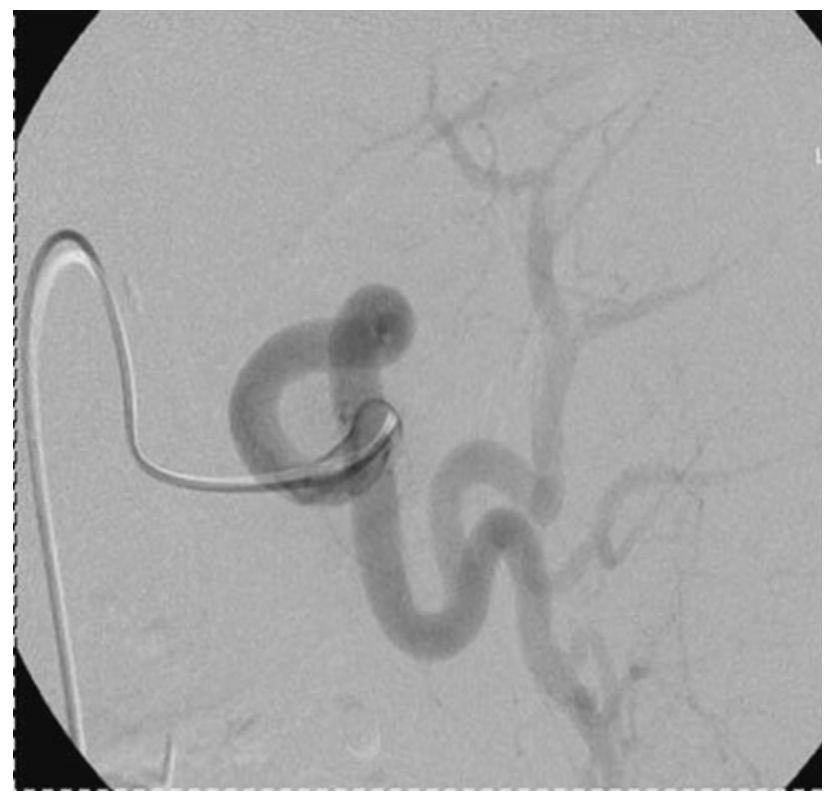

Fig. 2 Selective digital subtraction angiography of the splenic artery before embolization

At home, the patient developed progressive abdominal pain and weakness. Three days after SAE, the patient collapsed and was admitted to the emergency department. His temperature was $35.4^{\circ} \mathrm{C}$, blood pressure $65 / 45 \mathrm{~mm} \mathrm{Hg}$, pulse 105 beats/min, and respiratory rate 40 breaths/min. Abdominal examination revealed pain in the left upper quadrant, again without signs of peritonitis. The abdomen was hypotympana, and hardly any peristalsis was noted. Abnormal laboratory findings were as follows: thrombocytes $33 \times 10^{9} / \mathrm{L}$, leucocytes $34.7 \times 10^{9} / \mathrm{L}$, creatinine

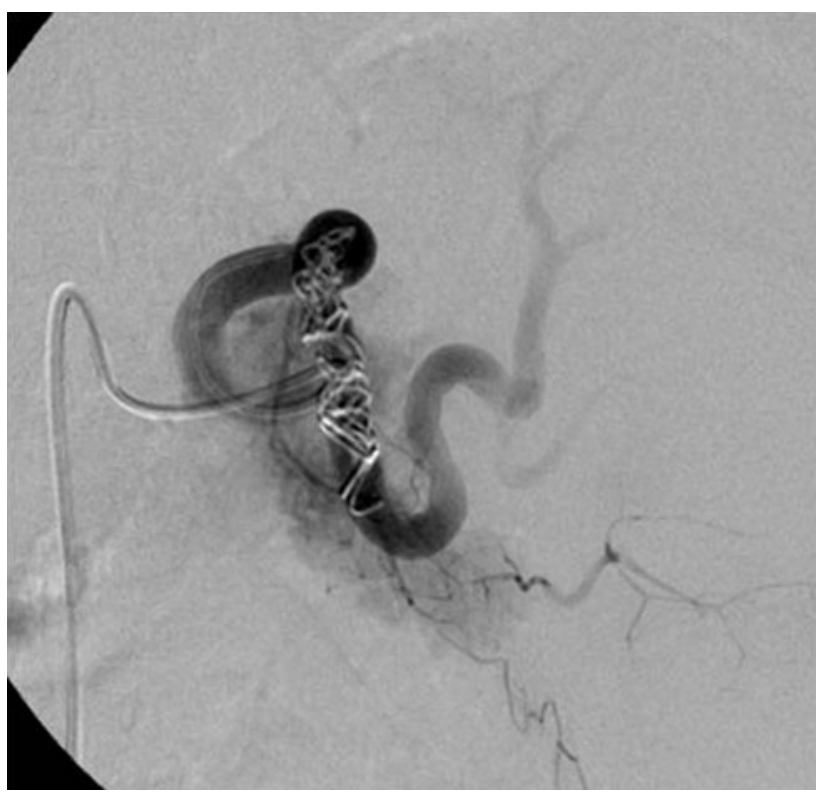

Fig. 3 Selective digital subtraction angiography of the splenic artery after embolization

$193 \mu \mathrm{mol} / \mathrm{L}$, lactate dehydrogenase $3285 \mathrm{mmol} / \mathrm{L}$, lactate

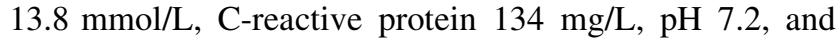
base excess -17 . In short, the patient exhibited hemodynamic shock with severe metabolic acidosis, renal failure, elevated infection parameters, and signs of severe necrosis.

Because of renal failure, a non-contrast-enhanced CT of the abdomen was performed. This revealed numerous small gas bubbles throughout the spleen consistent with extensive infarction (Fig. 4). There were no air-fluid levels or fluid collections suggestive for suppuration or abscess formation. In addition, there was no intra-abdominal free fluid or free air indicating bleeding or perforation. There were no signs indicating bowel ischemia, such as mural thickening, mesenteric fat stranding, intramural gas, nonobstructed bowel dilatation, or ascites [4-6]. A complementary ultrasound of the spleen showed multiple reverberation artifacts due to gas bubbles without signs of abscess formation $[7,8]$.

We hypothesized that the patient had severe sepsis or tumor lysis syndrome due to massive splenic infarction after embolization. The patient was rigorously rehydrated and subsequently received intravenous broad spectrum antibiotics and noradrenalin. Despite recovery of the circulation (blood pressure 118/50 mm Hg, pulse 93 beats/ $\mathrm{min}$ ), he developed progressive metabolic acidosis that did not react to intravenously administered sodium bicarbonate. Therefore, he was transferred to the operating room for a splenectomy. Laparotomy revealed extensive ischemia of the small bowel and colon with no therapeutic options. The patient was transferred to the intensive care department to receive palliative sedation. He died within several hours. 


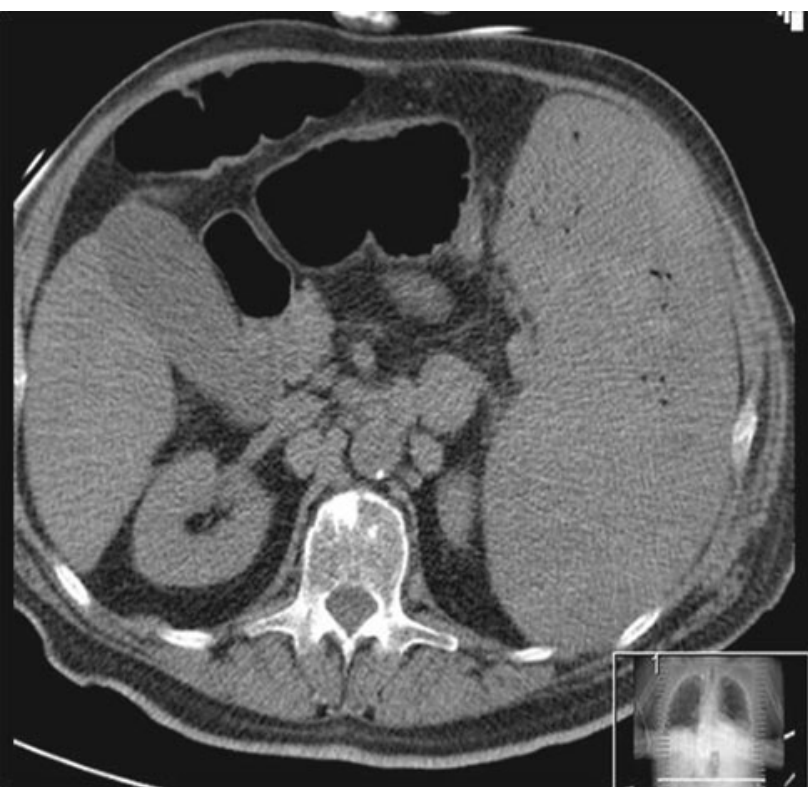

Fig. 4 Air bubbles are spread through entire spleen after embolization, a finding consistent with extensive infarction

At autopsy, severe ischemia of the small bowel and colon was confirmed, but no signs of arterial or venous obstruction were found in the mesenteric vessels. The spleen weighed $3945 \mathrm{~g}$ (the average normal weight is 150-200 g) and was completely avascular with coils in the splenic artery. There was no dislocation of coils noted. The pulmonary mass in the left lower lobe was confirmed to be an adenocarcinoma without metastasis. In addition, a pheochromocytoma was found in the right adrenal gland not previously identified at CT examination or suspected at clinical examination.

\section{Discussion}

SAE is widely accepted as nonsurgical management for blunt splenic trauma. It is also a well-recognized treatment for hypersplenism resulting in thrombocytopenia-for example, in patients with liver cirrhosis or hematological disorders. A relatively common complication of SAE is splenic infarction [1,9]. Some patients develop gas in the infarcted areas, although this is usually asymptomatic and does not necessarily implicate infection [7]. A few patients, however, develop infections and splenic abscesses, which are considered serious complications. Our hypothesis is that this patient developed a vaso-occlusive crisis caused by a pheochromocytoma triggered by the splenic embolization procedure. However, there were no blood catecholamine levels to support our theory. It was only after the autopsy that we discovered the presence of a pheochromocytoma.
To our knowledge, a fatal outcome after SAE due to a pheochromocytoma crisis has never been reported. A pheochromocytoma crisis is a rare life-threatening endocrine emergency with a high mortality rate. Acute and rapidly progressive hemodynamic disturbances result from the actions of high quantities of catecholamines secreted by the tumor and leading to organ failure. A pheochromocytoma crisis can present itself spontaneously or as a result of triggers such as trauma, surgery, anesthesia, and drug therapy. To our knowledge, there are no reports of a pheochromocytoma crisis specifically initiated by a SAE or any other embolization procedure.

Because no atherosclerosis or dislocated coils were discovered at autopsy in the mesenteric arteries, with exception of the splenic artery, the small bowel and colon necrosis most likely would have been the result of small vessel disease or hypovolemia. No signs of severe anemia or vasculitis were found. Therefore, we concluded that severe vasoconstriction was the probable cause. In patients with pheochromocytoma, a severe vaso-occlusive crisis, such as intense mesenteric artery vasoconstriction due to hypercatecholaminemia, is known to result in ischemic intestinal necrosis [10-12].

In conclusion, although a SAE is a well-known treatment for hypersplenism causing thrombocytopenia, one should be aware of serious postprocedural complications. Especially in massively enlarged spleens, potentially lethal complications can occur. In our case, a pheochromocytoma crisis triggered by SAE was the most likely cause of the described lethal complications.

Conflict of interest The authors declare that they have no conflict of interest.

Open Access This article is distributed under the terms of the Creative Commons Attribution Noncommercial License which permits any noncommercial use, distribution, and reproduction in any medium, provided the original author(s) and source are credited.

\section{References}

1. Madoff DC, Denys A, Wallace MJ et al (2005) Splenic arterial interventions: anatomy, indications, technical considerations, and potential complications. Radiographics 25(suppl 1):S191-S211

2. van der Vlies CH, Hoekstra J, Ponsen KJ et al (2011) Impact of splenic artery embolization on the success rate of nonoperative management for blunt splenic injury. Cardiovasc Intervent Radiol (in press)

3. Sabe AA, Claridge JA, Rosenblum DI et al (2009) The effects of splenic artery embolization on nonoperative management of blunt splenic injury: a 16-year experience. J Trauma 67:565-572

4. Horton KM, Fishman EK (2001) Multi-detector row CT of mesenteric ischemia: can it be done? Radiographics 21:1463-1473

5. Blachar A, Barnes S, Adam SZ et al (2011) Radiologists' performance in the diagnosis of acute intestinal ischemia, using 
MDCT and specific CT findings, using a variety of CT protocols. Emerg Radiol 18:385-394

6. Rha SE, Ha HK, Lee SH et al (2000) CT and MR imaging findings of bowel ischemia from various primary causes. Radiographics 20:29-42

7. Levy JM, Wasserman PI, Weiland DE (1981) Nonsuppurative gas formation in the spleen after transcatheter splenic infarction. Radiology 139:375-376

8. Killeen KL, Shanmuganathan K, Boyd-Kranis R (2001) CT findings after embolization for blunt splenic trauma. J Vasc Interv Radiol 12:209-214
9. Wu SC, Chen RJ, Yang AD (2008) Complications associated with embolization in the treatment of blunt splenic injury. World J Surg 32:476-482

10. Zacher LL, Teslow TW, Thompson RF (1989) Pheochromocytoma and ischemic enterocolitis. S D J Med 42:11-14

11. Szmulowicz UM, Savoie LM (2007) Ischemic colitis: an uncommon manifestation of pheochromocytoma. Am Surg 73: 400-403

12. Bravo EL, Gifford RW Jr (1993) Pheochromocytoma. Endocrinol Metab Clin North Am 22:329-341 